\title{
COBRA: Center-Oriented Broadcast Routing Algorithms for Wireless Ad Hoc Networks
}

\author{
Intae Kang and Radha Poovendran \\ Department of Electrical Engineering, University of Washington, Seattle, WA. 98195 \\ email: \{kangit,radha\}@ee.washington.edu
}

\begin{abstract}
In this paper we provide the initial framework for the study of center-oriented broadcast routing problems using omnidirectional antennas. From the intuition that the best place to take advantage of the wireless broadcast advantage is at the center of a network deploy region, we concretize this idea into a currently best performing power-efficient broadcast routing algorithm for wireless adhoc networks. We support this statement with extensive simulation studies.
\end{abstract}

\section{INTRODUCTION}

The broadcast routing problem over wireless ad hoc networks can be well modeled with a geometric (or proximity) graph theory [1], [2]. Especially, the most distinguishing property of the wireless medium called wireless broadcast advantage [3] is in fact very close to rephrasing the definition of a geometric graph, i.e., there exist edges for all node pairs if the distance between a pair is smaller than a given range constant (determined by the transmit power of each node). In other words, the wireless broadcast advantage property is indeed a geometric property. Therefore, finding a power-efficient broadcast routing tree for a given topology is almost tantamount to how fully exploited the underlying wireless broadcast advantage or geometric property of the specific node distribution.

Since broadcast with minimum total transmit power has been already proven to be NP-complete [4], developing efficient heuristics becomes even more important. Two of the most prominent heuristic algorithms called Broadcast Incremental Power (BIP) [3] and Embedded Wireless Multicast Advantage (EWMA) [4] represent the current state-of-the-art in terms of the effectiveness in utilizing the geometric properties. Because we are interested in enhancing the performance of algorithms for an ensemble of geometric graphs, some statistical or random geometric graph theoretic approach [1] may provide better understanding to the problem.

This paper is based on a very simple observation that the center of a deploy region is the best place to take advantage of the broadcast advantage property in a statistical sense, which was observed in our earlier work [5]. If the sum of the required power for unicasting from the source to a center and the transmit power for broadcasting from the center is smaller than the original broadcast routing tree rooted at the source node,

This research was funded in part by NSF grant ANI-0093187, ARO grant DAAD190210242 and Boeing grant 10198. this scheme fully makes sense. We will refer any algorithm implementing this scheme as a Center-Oriented Broadcast Routing Algorithm (COBRA) and the general scheme as a COBRA scheme. We do not know of any previous literature explicitly dealing with this scheme. Moreover, we take a further step to analyze this scheme: (i) From a geometrical point of view, we investigate how transmit power is wasted and analyze previously known algorithms. (ii) We present an analytical estimate of the expected minimum required power from the source to a center node. The derived expression seems to be robust over a large range of number of nodes. Anyone attempting to apply the center-oriented broadcast scheme may benefit from this result. (iii) Finally, we find an explicit algorithm outperforming all currently known algorithms to the best of our knowledge in terms of total transmit power.

The remainder of this paper is organized as follows: In the next section, by analyzing how power is wasted, we present some design principles that were the main motivation of this work. In Section III, we present analytical results that will be used in the next Section IV, where actual algorithms and simulation results are provided. Section $\mathrm{V}$ presents conclusions and summarizes our work.

\section{Geometric Analysis on the Source of Waste of TRANSMIT POWER}

A waste of power occurs when non-negligible amount of electromagnetic energy from omnidirectional antenna leaks into unwanted directions. While this is the fundamental reason, we further characterize the cause of waste of power in a greater detail from the geometrical point of view.

\section{A. Out of Boundary Power Loss}

Let's consider two broadcast routing trees over the same network topology illustrated in Fig. 1. $S$ represents the source node of a broadcast session. Note that the location of $S$ is different in each figure. Dashed lines represent the edges of broadcast routing tree, and the corresponding transmission ranges by omnidirectional antennas are drawn as circles. In this paper, we will identify the boundary of a specific topology as the convex hull of the particular node distribution, which is drawn with thick solid line in Fig. 1.

It is evident that any leakage of radiation power from an antenna out of the boundary is a waste of power, since there 


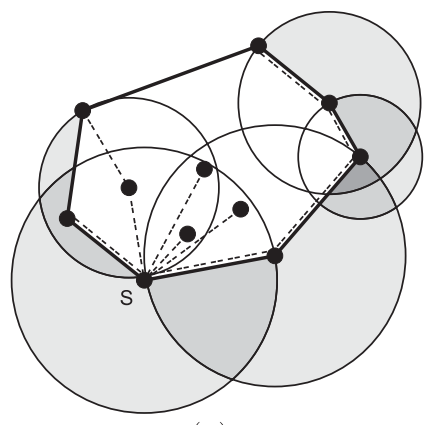

(a)

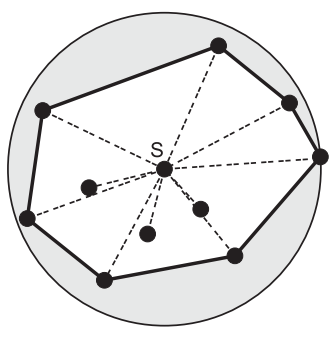

(b)
Fig. 1. Waste of power due to out of boundary power loss.

is no receiver of the broadcast traffic in that region. We will refer to this source of power waste as out of boundary power loss. The shaded region with gray corresponds to this loss. Clearly, the routing tree in Fig. 1(a) results in larger out of boundary power loss. Also, any darker colored region should be counted twice or more. The two scenarios in Fig. 1(a) and 1(b) exhibit different behavior. In Fig. 1(a), the source $S$ lies at one of the vertices of convex hull. Any transmission with large transmit power from a node near the boundary is guaranteed to be a waste of power. On the other hand, in Fig. 1(b), while $S$ transmits with even larger transmit power than the case in Fig. 1(a), out of boundary power loss is not much. So how can we mitigate this loss? We can achieve this only by assigning a small or no transmit power to the boundary nodes. This implies that power-efficient algorithms should be designed to satisfy, what we call, Conservative Outside Aggressive Inside (COAI) principle.

\section{B. Overlap Power Loss}

Now we consider another geometric source of power waste due to overlap of transmission ranges. Fig. 2 shows the sample examples of broadcast routing trees constructed with Minimumweight Spanning Tree (MST) and EWMA algorithm over $N=$ 20 randomly distributed nodes.

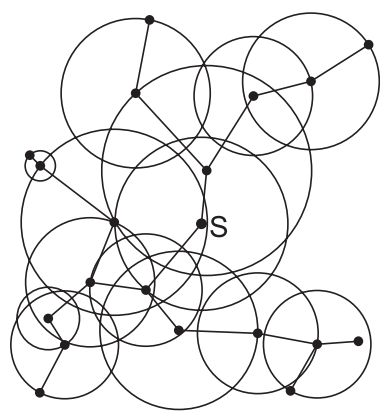

(a) MST tree

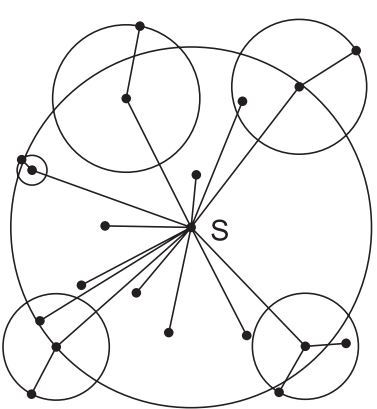

(b) EWMA tree
Fig. 2. Waste of power due to overlap power loss.

For every relay node, this overlap is inevitable because the messages should arrive from its parent node and the node also should retransmit the messages. It is a waste of power because the same region is covered more than once. We will refer to this source of power waste as overlap power loss. The effect of overlap power loss is quite evident in Fig. 2. In both Fig. 2(a) and 2(b), the power waste due to out of boundary power loss is almost the same-consider convex hulls in each figure-and is negligible. Nevertheless, the MST tree requires about $31 \%$ more transmit power due to overlap power loss. So how can we mitigate this effect? Because overlap is inevitable to relay traffic, the only way to reduce the effect is by reducing the number of overlaps, i.e., the number of relay nodes. We can achieve this by covering the whole region with a small number of large transmission ranges as EWMA and Greedy Perimeter Broadcast Efficiency (GPBE) [5] algorithms usually do.

The reliance on a small number of large transmit power has both advantages and disadvantages. First, this scheme is very effective at interference reduction, since nodes within the overlap region can receive the same message multiple times. Also, as shown in Fig. 2(b), nodes near the source $S$ enjoy a very large signal-to-noise ratio (SNR) and hence small bit error rate (BER). In addition, the average and maximum number of hops can be significantly reduced; the number of maximum hops of MST tree in Fig. 2(a) is 5, on the other hand, EWMA requires only two. Therefore, both strong SNR and small hop counts contribute significantly on the reliability of the trees and require much less retransmissions, which in turn further enhances energy-efficiency. However, this scheme is not favorable in every aspect. Since the scheme relies on a small number of nodes, unless effective load-balancing is implemented, the actual network lifetime can be shorter than other schemes. Hence, power-efficiency does not always translate to energyefficiency. For further details, interested readers are referred to our previous work [5]-[7].

\section{Analysis of Previous Algorithms}

In [6], [8], we introduced broadcast efficiency as a viable metric and demonstrated its effectiveness by developing broadcast routing algorithms called GPBE and S-GPBE, which is suitable for omnidirectional and directional (sector) antennas, respectively. The broadcast efficiency of a node is defined as the number of newly covered nodes per unit transmit power.

EWMA and GPBE are aggressive algorithms (meaning multiple nodes are included at the same time) and hence very effective at reducing overlap power loss. Also they are efficient at utilizing broadcast efficiency. However, while usually works well, there is no inherent protection mechanism to reduce the out of boundary power loss. On the other hand, MST and BIP are effective at reducing out of boundary power loss, because very small power is generally assigned to each node and these are the most conservative algorithms and only one node is added at each iteration. However, they are inefficient in terms of overlap power loss. While locally efficient in broadcast efficiency, their conservativeness causes a limited network view and the selected covers are not usually globally efficient in terms of broadcast efficiency. 


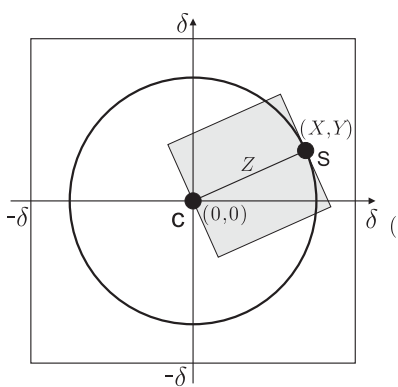

(a)

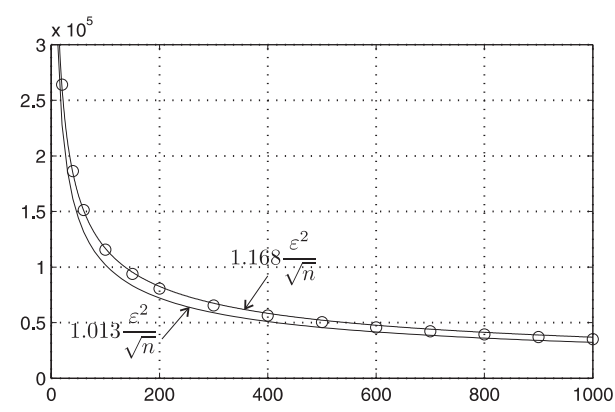

(c)

Fig. 3. (a) Mean distance to center node $C$ from source node $S$, (b) Illustration for approximate estimate of shortest path transmit power, (c) Estimation of expected transmit power of shortest path from $S$ to $C$.

Note that overlap power loss and out of boundary power loss is not always conflicting. In fact, we can achieve both as evidenced in Fig. 1(b) and Fig. 2(b) following COAI principle.

\section{ExPected Cost of Shortest PATH}

\section{A. Mean distance from a source node to center}

Let's consider the illustration in Fig. 3(a). In this paper, we assume a square deploy region $[-\delta, \delta] \times[-\delta, \delta]$, since many man-made structures usually impose rectilinear structures such as room, conference hall, street, and building walls, etc. We will use the path loss factor $\alpha=2$ throughout the paper. All nodes are randomly placed within the region following a spatial Poisson process with i.i.d. uniform distribution [2]. Let the coordinate of the source node $S$ be $(X, Y)$, where $X$ and $Y$ are i.i.d. uniform random variables with $|X| \leq \delta$ and $|Y| \leq \delta$. Let the distance from the source $S$ to center $C$ be another random variable $Z=\sqrt{X^{2}+Y^{2}}$, which is a function of $X$ and $Y$.

Considering boundary conditions, the cdf of $Z$ is given by

$$
\begin{aligned}
F_{Z}(z) & =\operatorname{Pr}\{Z \leq z\}=\operatorname{Pr}\left\{\sqrt{X^{2}+Y^{2}} \leq z\right\} \\
& = \begin{cases}\pi z^{2} /(2 \delta)^{2} & 0 \leq z<\delta \\
\left(\pi \delta^{2}+I_{1}(z)\right) /(2 \delta)^{2} & \delta \leq z<\sqrt{2} \delta \\
1 & z \geq \sqrt{2} \delta\end{cases}
\end{aligned}
$$

where

$$
\begin{aligned}
I_{1}(z) & =4 \int_{\delta}^{z} \int_{\cos ^{-1}(\delta / r)}^{\pi / 2-\cos ^{-1}(\delta / r)} r d \theta d r \\
& =\pi\left(z^{2}-\delta^{2}\right)+4 \delta \sqrt{z^{2}-\delta^{2}}-4 z^{2} \cos ^{-1}\left(\frac{\delta}{z}\right) .
\end{aligned}
$$

Since $Z$ is a non-negative random variable, the mean distance $E\{Z\}$ from source $S$ to center $C$ is

$$
\begin{aligned}
E\{Z\} & =\int_{0}^{\infty}\left[1-F_{Z}(z)\right] d z \\
& =\int_{0}^{\delta}\left(1-\frac{\pi z^{2}}{(2 \delta)^{2}}\right) d z+\int_{\delta}^{\sqrt{2} \delta}\left(1-\frac{\pi \delta^{2}+I_{1}(z)}{(2 \delta)^{2}}\right) d z \\
& =\frac{\sqrt{2}+\ln (1+\sqrt{2})}{3} \delta \approx 0.7652 \delta
\end{aligned}
$$

Fig. 3(a) shows the corresponding circle of radius $0.7652 \delta$.

\section{B. Approximate Estimation of Shortest Path Transmit Power}

Now let's consider the illustration in Fig. 3(b) which corresponds to the shaded square region in Fig. 3(a). Between $S$ and $C, n$ nodes are uniformly distributed within a $\varepsilon \times \varepsilon$ square region, where $S$ and $C$ lie at the center of each opposite edge. The square region is divided into $m$ equal strips. The more region (in terms of the number of strips) there exists in between the nodes, the better shortest path can be chosen, because there are more candidate paths to consider. For any randomly generated topology, we consider only the type of paths that pass through every node in a strip by increasing order of $x$ coordinates. We denote the required transmit power of the paths of this type as $P_{U}$ and that of the shortest path from $S$ to $C$ as $P \underset{S \rightarrow C}{S P T}$, where SPT denotes the shortest path tree. Because of the restriction of the path, $P \underset{S \rightarrow C}{S P T} \leq P_{U}$, and this holds regardless of specific instances. Hence, $E\left\{P_{S \rightarrow C}^{S P T}\right\} \leq E\left\{P_{U}\right\}$. We try to find the approximate expected value $E\{\underset{S \rightarrow C}{P P T}\}$ by minimizing the upper bound $E\left\{P_{U}\right\}$.

In the following, we only consider the strip containing $S$ and $C$, because the paths in other strips require more power on average. Since nodes are uniformly distributed, or produced by a spatial Poisson process, we assume $n / m$ nodes are inside the strip. For the following derivation, see Appendix for details.

For any $m$, we can minimize $E\left\{P_{U}\right\}$ so that

$$
\begin{aligned}
E\left\{P_{U}\right\} & \approx\left(\frac{n}{m}+1\right)\left[\left(\frac{\varepsilon}{3 m}\right)^{2}+\left(\frac{\varepsilon}{n / m+1}\right)^{2}\right] \\
& \approx\left(\frac{n}{9 m^{3}}+\frac{m}{n}\right) \varepsilon^{2},
\end{aligned}
$$

where we assume $n / m \gg 1$ at the second step. (See Appendix for proof.) To find the minimum value of $E\left\{P_{U}\right\}$, we calculate

$$
\frac{d}{d m} E\left\{P_{U}\right\}=\left(-\frac{n}{3 m^{4}}+\frac{1}{n}\right) \varepsilon^{2}=0 .
$$

Therefore the minimum value is attained when $3 m^{4}=n^{2}$, i.e., $m=\frac{1}{\sqrt[4]{3}} \sqrt{n}$ and the minimum value is

$$
E\{P \underset{S \rightarrow C}{S P T}\} \approx \frac{4}{3 \sqrt[4]{3}} \frac{\varepsilon^{2}}{\sqrt{n}}=\eta \frac{\varepsilon^{2}}{\sqrt{n}}, \quad \eta=1.013 .
$$

The most notable thing is that the upper bound of the transmit power of the shortest path from $S$ to $C$ is proportional to $1 / \sqrt{n}$ of the area $\varepsilon^{2}$. Hence, the more nodes are inside the square region, the less transmit power is required. 
To verify how well this equation (4) fits with actual situations, we performed a simulation study. For each network size $N$, we generated 1000 random topologies and calculated the required transmit power of the shortest path from $S$ to $C$. We found the proportionality constant $\eta=1.013$ in (4) is somewhat optimistic leading to smaller average values. This is partially due to the two approximation steps in our derivation. Nevertheless, we could confirm the dependence of on $\varepsilon^{2} / \sqrt{n}$ as shown in Fig. 3(b). Using least square regression, the constant $\eta=1.168$ with $95 \%$ confidence bounds $(1.156,1.179)$ matches better for actual simulation runs. We suggest using this value instead of (4), as it gives more conservative bounds.

\section{Algorithm Description}

The basic idea of a center-oriented broadcast routing algorithm (COBRA) is that sources having broadcast messages send the message to a center of deploy region with smallest possible transmit power and let the center node relay and broadcast the message. In any COBRA scheme, the following three components should be well-defined and clearly specified:

1) the definition of a center node $C$.

2) a unicast path from source $S$ of broadcast to $C$.

3 ) the broadcast routing algorithm from the center node.

Further discussion on the elaborate definition of a center will be presented in Section IV-D. We start from the simplest schemes in the following section. Depending on the used central broadcast algorithm, we specify the algorithm as a suffix to COBRA.

\section{A. Scheme 1: A Naive COBRA-MAX Algorithm}

Following the previous argument, the simplest conceivable scheme is source $S$ transmits messages to center $C$ along the minimum power shortest path. Recall that in this paper we exclusively use the square deploy region $[-\delta, \delta] \times[-\delta, \delta]$. We assume the path loss factor $\alpha=2$. The following analysis can be easily extended to other values of $\alpha$. For now, we assume that there always exists a center node with coordinate $\left(x_{C}, y_{C}\right)$ located at $(0,0)$. In the first approach, source $S$ transmits to $C$ along the minimum power shortest path and node $C$ transmits with maximum power $(\sqrt{2} \delta)^{2}$ to cover the whole deploy region. Thus, we refer to this scheme as COBRA-MAX algorithm. The advantage of this approach is that center $C$ does not require any location or distance information of other nodes. Clearly, this is not a power-efficient scheme, because there is much power waste due to out of boundary power loss. Using the previous derivation (1) and (4) with $\eta=1.168$, setting $\varepsilon=0.7652 \delta$ and $n=N \varepsilon^{2} /(2 \delta)^{2}$, the approximate expected total transmit power of this tree is:

$$
E\left\{\mathcal{P}\left(T_{\text {COBRA }}\right)\right\}=2 \delta^{2}+1.168 \frac{\varepsilon^{2}}{\sqrt{N \frac{\varepsilon^{2}}{(2 \delta)^{2}}}}=\left(2+\frac{1.788}{\sqrt{N}}\right) \delta^{2}
$$

As a second approach, we can reduce the transmit power from center $C$ by transmitting only up to the farthest node.
This requires that the center node collect distance information from itself to every node in the network. Then, the approximate expected total transmit power is:

$$
E\left\{\mathcal{P}\left(T_{\text {COBRA }}\right)\right\}=E\left\{\max _{j \in N \backslash\{S \rightarrow C\}} d_{C j}^{2}\right\}+\frac{1.788}{\sqrt{N}} \delta^{2}
$$

where $\{S \rightarrow C\}$ denotes the set of nodes lying on the shortest path from $S$ to $C$.

\section{B. Scheme 2: COBRA-EWMA Algorithm}

We denote a node closest to the center of deploy region $(0,0)$ as a center node $C$, i.e.,

$$
C=\underset{i \in N}{\arg \min } \sqrt{x_{i}^{2}+y_{i}^{2}}
$$

To choose a right broadcast routing algorithm from the center node, we rely on simulation results rather than theoretical analysis. What we want is the best performing algorithm when the source is located at the center. The data used here were readily available from our previous work [5], [6]. We tested the performance of four algorithms including EWMA, GPBE, $\mathrm{BIP}$, and MST. The ratio of total transmit power from random source location and from the center was obtained for each given topology, and the average value for 100 difference topologies are calculated and listed in Table 1.

TABLE I

THE RATIO OF TOTAL TRANSMIT POWER FROM RANDOM SOURCE LOCATION AND FROM THE CENTER $(\alpha=2)$.

\begin{tabular}{|l|c|c|c|c|c|c|c|}
\hline \multicolumn{1}{|c|}{$\mathrm{N}$} & 20 & 40 & 60 & 100 & 150 & 200 & 300 \\
\hline \hline EWMA & 1.2473 & 1.1991 & 1.1825 & 1.1667 & 1.1610 & 1.1558 & 1.1585 \\
\hline GPBE & 1.2177 & 1.1345 & 1.1375 & 1.1138 & 1.1070 & 1.0906 & 1.0861 \\
\hline BIP & 1.0498 & 1.0125 & 1.0003 & 1.0003 & 1.0002 & 1.0004 & 1.0000 \\
\hline MST & 1.0108 & 1.0062 & 0.9993 & 1.0028 & 1.0011 & 1.0000 & 0.9996 \\
\hline
\end{tabular}

Table 1 demonstrates that the choice of source location greatly impacts the performance of EWMA and GPBE algorithms. Remarkably, about 16 25\% for EWMA and 9 22\% for GPBE reduction in power $(\Delta P)$ is observable. Note that this is significant savings in power consumption considering that BIP algorithm, which is the most well-known algorithm for this purpose, produces about $7 \%$ reduction in total transmit power from MST [3]. ${ }^{1}$ On the other hand, both BIP and MST are not affected by the source location and hence there is no point using these algorithms for center-based broadcast scheme. Thus, the choice of algorithm is obvious, EWMA, because of its good performance as a central broadcast algorithm. Although we use EWMA here, the underlying concept is completely different. Also, note that COBRA scheme is not limited to a specific algorithm.

The final remaining choice is the unicast routing algorithm from the original source of broadcast to the center node defined

\footnotetext{
${ }^{1}$ This statement is based on our simulation studies. This value corresponds to the case when $|N| \geq 150$. The reason for choosing this value is that we believe the transient behavior due to the effect of node density seems to be filtered out after this range and this value represents a reasonable estimate of a steady state behavior.
} 


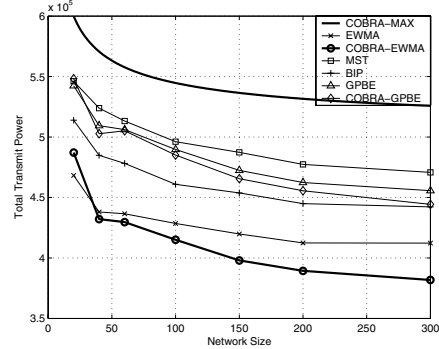

(a)

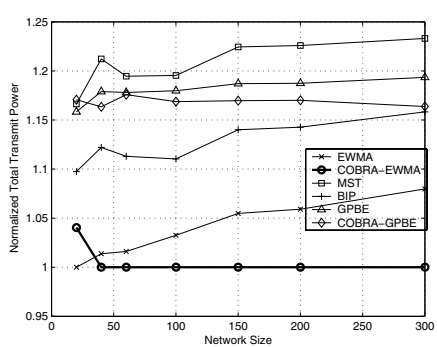

(b)
Fig. 4. Comparison of various algorithms in terms of (a) total transmit power and (b) normalized total transmit power. $(\alpha=2)$

above. While it may be possible to choose any unicast routing algorithm to satisfy certain other requirements such as loadbalancing, throughput or delay, we simply use the shortest path tree (SPT) algorithms such as Dijkstra or distributed BellmanFord algorithm [9] using the transmit power as the cost of each link between the nodes.

Combining all these factors, what remains to be seen is whether the power from $S$ to $C$ is smaller than the savings presented in Table 1, i.e., $\Delta P>E\left\{P_{S \rightarrow C}^{S P T}\right\} \approx \eta \frac{\varepsilon^{2}}{\sqrt{n}}$. Before we proceed to a simulation study, we first confirmed that this relation really holds at least on average.

\section{Simulation Results}

We compared several algorithms including EWMA, GPBE, BIP, MST, COBRA-EWMA, and COBRA-GPBE, where we used both EWMA and GPBE as central broadcast algorithms, since large gains are exhibited in Table 1. Path loss factor $\alpha=$ 2 is used. Fig. 4 is the summary of our simulation results. Each point in Fig. 4 corresponds to an average value over 100 different randomly generated topologies.

Fig. 4(a) presents the performance comparison in terms of total transmit power as a function of network size $N$ per square deploy region with $\delta=500 \mathrm{~m}$. The curve corresponding to COBRA-MAX (see eqn. (5)) is drawn with a thick solid line for comparison. In general, as $N$ becomes larger, the required total transmit power of all algorithms reduces. We can observe that COBRA-EWMA algorithm outperforms EWMA, except at $N=20$, and all other algorithms for every network size. This is because the cost of the shortest path exceeds the benefit of COBRA scheme for small $N$. The separation between curves of COBRA-EWMA and EWMA gets even larger as $N$ grows. Consequently, COBRA-EWMA provides the best performance in terms of total transmit power.

To facilitate easy comparison with previous work [3]-[5], we also present in Fig. 4(b) the results in terms of the normalized total transmit power as a metric:

$$
\mathcal{P}_{T X}^{\text {norm }}\left(T_{\text {algorithm }}\right)=\frac{\mathcal{P}_{T X}\left(T_{\text {algorithm }}\right)}{\min _{i \in \text { algorithm }}\left\{\mathcal{P}_{T X}\left(T_{i}\right)\right\}} .
$$

In contrast to Fig. 5 in [5] where the curves were relatively flat, the curves in Fig. 4(b) tend to increase leading to even larger separation between COBRA-EWMA and the rest of the algorithms as $N$ grows. This figure reconfirms the superior performance of COBRA-EWMA algorithm. It is left as our future work to verify this tendency in much larger network sizes than $N=300$.

In summary, up to now, BIP [3] has contributed about $7 \%$ reduction in total transmit power over MST, and EWMA [4] has contributed about $16 \%$ reduction over MST. We introduced in this paper a general scheme based on center-oriented broadcast and presented another algorithm giving up to $23 \%$ reduction over MST and hence currently the best performing algorithm as of now. If the difference in power shown in Table 1 persists for large $N$, the separation over EWMA will be larger (say $15 \%$ as in the last column of Table 1), because shortest path cost becomes negligible as $N \rightarrow \infty$ without considering processing and reception costs.

\section{Other Considerations and Future Work}

Note that in previous section even with a simple definition of the center node, we still got very favorable performance results. For a fixed deploy region imposed by physical surroundings such as walls or room structure, this definition is not an unreasonable choice at all. However, we believe that, for each specific topology, a more elaborate definition of the center node can provide further reduction in transmit power. For example, a center of mass or the smallest bounding circle centered at node $C=\min _{i \in N}\left\{\max _{j \in N}\left\{d_{i j}\right\}\right\}$ may give better results. Further refinement on the definition of center and the analysis of its effect on overall performance are reserved as our future work.

Alternatively, as an extreme case, we can try every node as a center of broadcast with complexity multiplied by $N$. This approach is guaranteed to provide better performance than the current one. The time complexity of EWMA is given by $O\left(d^{4}\right) m^{2}$, where $d$ denotes the maximum node degree and $m$ denotes the total number of transmitting nodes [4]. Thus using the exhaustive scheme, the complexity becomes $O\left(N d^{4}\right) m^{2}+O(N \log N+E)$ where $E$ denote the number of edges, because we need to run SPT algorithm only once to get the shortest path tree from $S$ to all nodes.

We can think of other strategies to improve the performance. For instance, the center node need not broadcast to the nodes lying on the unicast path from the source. Merging this effect into the algorithm will give better performance especially for small network sizes. In addition, whether an algorithm is distributable is an important scalability issue. Since distributed versions of SPT [9] and EWMA [4] are known, if we can distribute the center election algorithm, the full process becomes distributable. We intend to study the center election algorithm to make COBRA fully distributed.

\section{CONCLUSIONS}

In this paper, we presented a center-oriented broadcast routing (COBRA) scheme. While it is a simple conceptual extension, we demonstrated that this leads to the currently best performing broadcast routing algorithm. Of course, its superior 
performance is largely indebted to the effectiveness of EWMA for use as a central broadcast algorithm. However, we can eventually use any algorithm that will be developed in the future specifically targeted at enhancing the performance from the center node, because broadcast only from the center can greatly simplify the complexity of design principles we considered. We consider there are still further room for improvement and the breakthrough should come from the better understanding of the underlying geometric and statistical properties.

\section{REFERENCES}

[1] M. Penrose, Random Geometric Graphs. Oxford University Press, 2003.

[2] R. C. Larson and A. R. Odoni, Urban Operations Research. Englewood Cliffs, N.J.: Prentice-Hall, Inc., 1981.

[3] J. E. Wieselthier, G. D. Nguyen, and A. Ephremides, "On the construction of energy-efficient broadcast and multicast trees in wireless networks," in Proc. IEEE INFOCOM 2000, vol. 2, 2000, pp. 585-94.

[4] M. Cagalj, J. P. Hubaux, and C. Enz, "Minimum-energy broadcast in all-wireless networks: Np-completeness and distribution issues," in Proc ACM/IEEE MOBICOM '02, Atlanta, Georgia, 2002.

[5] I. Kang and R. Poovendran, "A novel power-efficient broadcast routing algorithm exploiting broadcast efficiency," in IEEE Vehicular Technology Conference (VTC), Orlando, FL, 2003.

[6] - "A comparison of power-efficient broadcast routing algorithms," in IEEE GLOBECOM 2003, San Francisco, CA, 2003.

[7] — "Maximizing static network lifetime of wireless broadcast adhoc networks," in Proc. IEEE ICC 2003, Anchorage, Alaska, 2003.

[8] — - "S-GPBE: a power-efficient broadcast routing algorithm using sectored antenna," in Proc. IASTED WOC '03, Banff, Alberta, Canada, 2003.

[9] T. H. Cormen, C. E. Leiserson, R. L. Rivest, and C. Stein, Introduction to Algorithms, 2nd ed. Cambridge, Mass.: MIT Press, 2001.

[10] S. M. Ross, Stochastic Processes, 2nd ed. John Wiley Sons, 1995.

\section{APPENDIX}

\section{A. Proof of Equation (2)}

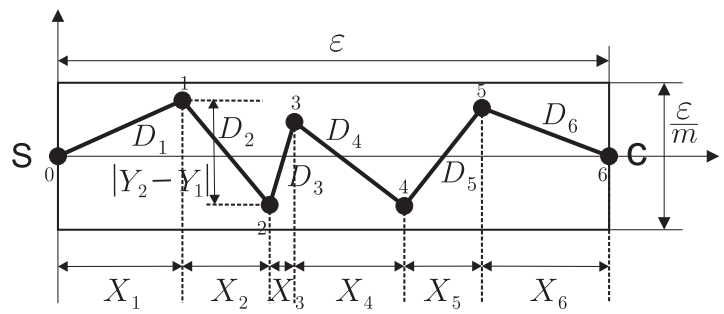

Fig. 5. Mean distance to center node $C$ from source node $S$

Let's consider Fig. 5 where $n / m$ nodes are randomly distributed within a strip of size $\varepsilon \times \varepsilon / m$. Let $\alpha=2$. Node 0 corresponds to $S$ and $C$ is node $(n / m+1)$. The coordinate of node $i$ is defined by a pair of random variables $\left(S_{i}, Y_{i}\right)$, $S_{i}=\sum_{k=0}^{i} X_{k}$, where we interpret $S_{i}$ and $X_{i}$ as an epoch and interarrival variable, respectively [10]. We denote a random variable corresponding to the total transmit power of the path which pass through every node in the strip in order by $x$ coordinate as $P_{U}$. A random variable $D_{i}$ corresponding to distance between node $(i-1)$ and $i$ satisfies $D_{i}^{2}=X_{i}^{2}+\left(Y_{i}-Y_{i-1}\right)^{2}$ for all $1 \leq i \leq \frac{n}{m}+1$. Then, $P_{U}$ can be expressed as

$$
P_{U}=\sum_{i=1}^{n / m+1} D_{i}^{2}=\sum_{i=1}^{n / m+1}\left(X_{i}^{2}+\left(Y_{i}-Y_{i-1}\right)^{2}\right) .
$$

Taking expectation at both sides,

$$
\begin{aligned}
E\left\{P_{U}\right\} & =E\left\{\sum_{i=1}^{n / m+1}\left(X_{i}^{2}+\left(Y_{i}-Y_{i-1}\right)^{2}\right)\right\} \\
& =\sum_{i=1}^{n / m+1}\left(E\left\{X_{i}^{2}\right\}+E\left\{\left(Y_{i}-Y_{i-1}\right)^{2}\right\}\right) \\
& \geq \sum_{i=1}^{n / m+1}\left(E^{2}\left\{X_{i}\right\}+E^{2}\left\{\left|Y_{i}-Y_{i-1}\right|\right\}\right) .
\end{aligned}
$$

where in the third step, we used Jensen's inequality [10] because $f(x)=x^{2}$ is a convex function.

Now we consider $x$-axis and $y$-axis separately. We consider $x$ coordinate first. Because the nodes are distributed according to spatial Poisson process $N(x), X_{i}, 1 \leq i \leq n / m$, is exponentially distributed. From the theory of random process [10], using order statistics, given that $n / m$ events has occurred in the interval $(0, \varepsilon)$, the unordered random variables are considered to be distributed independently and uniformly. Note that we implicitly assumed the condition $N(\varepsilon)=n / m$. Hence, this results in

$$
E\left\{X_{i}\right\}=E\left\{X_{i} \mid N(\varepsilon)=\frac{n}{m}\right\}=\frac{\varepsilon}{(n / m+1)} .
$$

For $y$ coordinate, we are only interested in mean distance between two adjacent points $Y_{i}$ and $Y_{i+1}$ which are uniformly and independently distributed. Let $Y=\left|Y_{i}-Y_{i+1}\right|$ where $-\frac{\varepsilon}{2 m} \leq Y_{i}, Y_{i+1} \leq \frac{\varepsilon}{2 m}$ and $0 \leq Y \leq \frac{\varepsilon}{2 m}$. Then the cdf $F_{Y}(y)$ is

$$
\begin{aligned}
F_{Y}(y) & =\operatorname{Pr}\{Y \leq y\}=\operatorname{Pr}\left\{\left|Y_{i}-Y_{i+1}\right| \leq y\right\} \\
& =\left[\left(\frac{\varepsilon}{m}\right)^{2}-\left(\frac{\varepsilon}{m}-y\right)^{2}\right]\left(\frac{m}{\varepsilon}\right)^{2}=2 \frac{m}{\varepsilon} y-\frac{m^{2}}{\varepsilon} y^{2}
\end{aligned}
$$

for $0 \leq y \leq 1 / m$, and $F_{Y}(y)=1$, otherwise. Therefore,

$$
\begin{aligned}
E\{Y\} & =\int_{0}^{\infty}\left[1-F_{Y}(y)\right] d y=\int_{0}^{\frac{1}{m}}\left(1-2 \frac{m}{\varepsilon} y+\frac{m^{2}}{\varepsilon} y^{2}\right) d y \\
& =y-\frac{m}{\varepsilon} y^{2}+\left.\frac{\left(\frac{m}{\varepsilon}\right)^{2} y^{3}}{3}\right|_{0} ^{\varepsilon / m}=\frac{\varepsilon}{3 m}
\end{aligned}
$$

In fact, we can derive the same result using exactly the same argument as (9) using different parameter values such that

$$
E\{Y\}=E\left\{Y \mid N\left(\frac{\varepsilon}{m}\right)=2\right\}=\frac{\varepsilon / m}{(2+1)}=\frac{\varepsilon}{3 m} .
$$

Therefore replacing (9) and (10) into (8), we can minimize $E\left\{P_{U}\right\}$ as

$$
\begin{aligned}
E\left\{P_{U}\right\} & \geq \sum_{i=1}^{n / m+1}\left[\left(\frac{\varepsilon}{n / m+1}\right)^{2}+\left(\frac{\varepsilon}{3 m}\right)^{2}\right] \\
& =\left(\frac{n}{m}+1\right)\left[\left(\frac{\varepsilon}{n / m+1}\right)^{2}+\left(\frac{\varepsilon}{3 m}\right)^{2}\right]
\end{aligned}
$$

which corresponds to (2). 